\title{
Estratégias de prevenção do suicídio e da autolesão voltadas para adolescentes em ambientes escolares: uma revisão integrativa da literatura
}

\author{
Suicide and self-injury prevention strategies for adolescents in school settings: an integrative \\ literature review \\ Estrategias de prevención del suicidio y las autolesiones para adolescentes en el ámbito escolar: una \\ revisión bibliográfica integradora
}

Recebido: 25/01/2022 | Revisado: 01/02/2022 | Aceito: 06/02/2022 | Publicado: 07/02/2022

\author{
Amanda de Morais Pinto Ribeiro Escobar \\ ORCID: https://orcid.org/0000-0002-9009-1529 \\ Universidade de Pernambuco, Brasil \\ E-mail: amanda.morais@upe.br \\ Mariana de Fátima Alves Arruda \\ ORCID: https://orcid.org/0000-0002-9537-4405 \\ Universidade de Pernambuco, Brasil \\ E-mail:mariana.arruda@upe.br \\ José Eudes de Lorena Sobrinho \\ ORCID: https://orcid.org/0000-0001-7820-735X \\ Universidade de Pernambuco, Brasil \\ E-mail: eudes.lorena@upe.br
}

\begin{abstract}
Resumo
Os comportamentos suicidas e de autolesão são um desafio para a Saúde Pública, especificamente para a saúde do adolescente, necessitando de intervenções intersetoriais. Neste sentido, os ambientes escolares mostram-se como espaços potenciais para o desenvolvimento de estratégias de prevenção dos citados comportamentos. O objetivo deste estudo foi analisar na literatura estratégias eficazes de prevenção do suicídio e da autolesão voltadas para adolescentes em ambientes escolares. Trata-se de uma revisão integrativa da literatura, realizada no período de setembro a outubro de 2021, com a utilização das seguintes bases de dados online: Medical Literature Analysis and Retrievel System Online (MEDLINE), Literatura Latino-americana e do Caribe em Ciências da Saúde (LILACS), Índice Bibliográfico Español en Ciências de La Salud (IBECS), Base de Dados em Enfermagem (BDENF), Coleciona SUS e Index Psicologia- Periódicos pesquisadas via portal da Biblioteca Virtual em Saúde (BVS) e na base Education Resources Information Center (ERIC). Ao realizar as etapas previstas na revisão resultaram um total de 11 artigos que atenderam aos critérios de elegibilidade do estudo. As intervenções de prevenção ao suicídio e da autolesão encontradas nesta revisão de literatura abordaram questões de saúde mental através do fortalecimento das habilidades socioemocionais e os programas apontaram predominância de treinamento para reconhecimento dos sinais do comportamento suicida e encaminhamento aos profissionais da saúde mental, principalmente o Gatekeeper (guardiões da vida). Quanto à eficácia dos estudos incluídos, foi verificado que existiram resultados promissores na maioria deles, entretanto alguns destes apresentaram lacunas.
\end{abstract}

Palavras-chave: Adolescentes; Suicídio; Autolesão; Prevenção; Escola.

\begin{abstract}
Suicidal and self-harm behaviors are a challenge for Public Health, specifically for adolescent health, requiring intersectoral interventions. In this sense, school environments are potential spaces for the development of prevention strategies for the aforementioned behaviors. The objective of this study was to analyze in the literature effective suicide and self-injury prevention strategies aimed at adolescents in school environments. This is an integrative literature review, carried out from September to October 2021, using the following online databases: Medical Literature Analysis and Retrievel System Online (MEDLINE), Latin American and Caribbean Literature in Science of Health (LILACS), Bibliographic Index Español en Ciências de La Salud (IBECS), Database on Nursing (BDENF), SUS Collection and Psychology Index- Periodicals searched via the Virtual Health Library (BVS) portal and the Education Resources database Information Center (ERIC). When carrying out the steps foreseen in the review, a total of 11 articles resulted that met the eligibility criteria of the study. The suicide and self-harm prevention interventions found in this literature review addressed mental health issues through the strengthening of socio-emotional skills and the programs indicated a predominance of training to recognize the signs of suicidal behavior and referral to mental health professionals, especially the Gatekeeper (guardians of life). Regarding the effectiveness of the included studies, it was verified that there were promising results in most of them, however some of them showed a gaps.
\end{abstract}

Keywords: Adolescents; Suicide; Self-injury; Prevention; School. 


\begin{abstract}
Resumen
Las conductas suicidas y autolesivas constituyen un desafío para la Salud Pública, específicamente para la salud de los adolescentes, que requiere intervenciones intersectoriales. En este sentido, los ambientes escolares son espacios potenciales para el desarrollo de estrategias de prevención de las conductas mencionadas. El objetivo de este estudio fue analizar en la literatura estrategias efectivas de prevención del suicidio y las autolesiones dirigidas a adolescentes en ambientes escolares. Esta es una revisión integradora de la literatura, realizada de septiembre a octubre de 2021, utilizando las siguientes bases de datos en línea: Medical Literature Analysis and Retrievel System Online (MEDLINE), Latin American and Caribbean Literature in Science of Health (LILACS), Bibliographic Index Español en Ciências de La Salud (IBECS), Base de Datos de Enfermería (BDENF), Colección SUS e Índice de PsicologíaPeriódicos buscados a través del portal Biblioteca Virtual en Salud (BVS) y el Centro de Información de la base de datos de Recursos Educativos (ERIC). Al realizar los pasos previstos en la revisión resultaron un total de 11 artículos que cumplieron con los criterios de elegibilidad del estudio. Las intervenciones de prevención del suicidio y las autoagresiones encontradas en esta revisión de la literatura abordaron problemas de salud mental a través del fortalecimiento de habilidades socioemocionales y los programas indicaron un predominio de la capacitación para reconocer los signos de la conducta suicida y la derivación a profesionales de la salud mental, especialmente al Gatekeeper (guardianes de la vida). En cuanto a la efectividad de los estudios incluidos, se verificó que hubo resultados prometedores en la mayoría de ellos, sin embargo, algunos de ellos mostraron unas brechas.
\end{abstract}

Palabras clave: Adolescentes; Suicidio; Autolesiones; Prevención; Escuela.

\title{
1. Introdução
}

Segundo a Organização Mundial da Saúde (OMS) os comportamentos suicidas incluem o pensar em suicídio (ou ideação), o planejamento do ato, a tentativa e o próprio suicídio, ao passo que a autolesão é definida como qualquer comportamento intencional envolvendo agressão direta ao próprio corpo sem a intenção suicida consciente (WHO, 2014; Giusti, 2013).

Neste sentido, o suicídio envolve não tanto o desejo de matar o corpo, mas o desejo para acabar com a própria consciência. Enquanto, a autolesão é um problema emocional caracterizado por comportamentos propositais que envolvem agressões diretas ao próprio corpo, as lesões são quase sempre superficiais e geralmente feitas em pontos de fácil acesso, como braços, pernas, tórax e abdômen. As técnicas mais comuns são cortes com estiletes, giletes ou tesouras, queimaduras de cigarro, arranhões e mordidas (Aratangy, et al., 2018). Os adolescentes e jovens que praticam autolesão apresentam uma probabilidade três vezes maior de terem comportamentos suicidas (Halicka \& Kiejna, 2018).

O suicídio representa $1,4 \%$ de todas as mortes no mundo, tornando-se, em 2012, a $15^{\text {a }}$ causa de mortalidade na população geral e a segunda entre os jovens de 15 a 29 anos. Estes números demonstram um cenário crítico, visto que foi estabelecida uma meta a nível mundial, para ser alcançada até o ano de 2020, de diminuição em $10 \%$ os casos, no entanto não houve concretização deste acordo (WHO, 2014; Silva, 2019). Os estudos sobre prevalência da autolesão na população mundial têm resultados bastante variados, há tendências de serem mais prevalentes em adolescentes e existem registros de aumento nos últimos anos (Giusti, 2013).

Nesta circunstância, durante o período da adolescência as competências relacionadas às questões socioemocionais apresentam grande importância para a vida saudável, tendo como base o apoio da família, da escola e da comunidade (WHO, 2018). Ao iniciar este período, é imprescindível que pessoas responsáveis pelos adolescentes e os profissionais de saúde concedam o suporte necessário, com o objetivo de mitigar condutas que levem ao risco nesta faixa etária (Carroll et al., 2016), uma vez que trata-se de uma fase de busca de autonomia e autoconhecimento, sendo marcada por conflitos internos e externos (Quesada et al., 2020). Logo, a adolescência é um fenômeno complexo e produto da interação entre processos do desenvolvimento biológico e sociocultural (Fernandes \& Santos, 2020).

É nesse contexto que emergem os comportamentos suicidas e de autolesão, que se trata de um problema de saúde pública significativo para adolescentes, onde os fatores culturais como gênero, raça e pobreza podem colocá-los em maior vulnerabilidade (Tomek et al., 2018). Alguns dos fatores de risco para os referidos comportamentos são: vivência de 
violências, conflitos familiares, uso de substâncias psicoativas, pouco suporte social, decepção amorosa, solidão, histórico familiar de comportamento suicida dentre outros (Sousa, et al., 2020).

À vista disso, desde décadas passadas, a Organização Mundial da Saúde (OMS) vem compreendendo a necessidade da instauração de uma política de monitoramento dos casos de suicídio e a consequente realização de ações focadas na prevenção deste e na promoção de saúde mental (WHO, 2014). Neste sentido, o vínculo entre a escola e os adolescentes são primordiais para o desenvolvimento de estratégias de prevenção aos comportamentos suicidas e de autolesão. Portanto, é essencial que sejam ofertadas nos ambientes escolares para os pais, cuidadores e profissionais da educação, intervenções que sejam capazes de identificar os primeiros sinais de comportamentos suicidas, a fim de fornecer o apoio necessário aos adolescentes (Hedeland et al., 2016).

Diante do exposto, a presente pesquisa teve como objetivo analisar na literatura estratégias eficazes de prevenção do suicidio e da autolesão voltadas para adolescentes em ambientes escolares. A importância do estudo consiste em somar dados científicos sobre o assunto que se trata de um tema relevante para saúde pública com interface na educação.

\section{Metodologia}

Trata-se de uma revisão integrativa da literatura, que é definida como um método de realização de pesquisa através da Prática Baseada em Evidências (PBE). Este tipo de revisão tem a função de detectar limitações presentes nos estudos, a partir da busca de artigos nas bases de dados, para então desenvolver pesquisas com a finalidade de responder às lacunas vigentes (Souza, Silva \& Carvalho, 2010).

Para elaboração desta revisão integrativa, foram realizadas as seguintes etapas: identificação do tema a ser estudado e a pergunta de pesquisa; construção dos critérios de elegibilidade (inclusão e exclusão) e posterior busca de publicações nas bases de dados online; estabelecimento do conteúdo que será extraído e sumarizado após a análise dos artigos e a categorização destes; avaliar os artigos que foram incluídos através dos critérios utilizados; elaboração dos resultados e interpretá-los; finalização e apresentação da revisão integrativa da literatura.

A pergunta condutora dessa revisão integrativa foi construída por meio da estratégia PICO, sendo considerada população, fenômeno de interesse e contexto, resultando no seguinte texto: Quais as principais estratégias eficazes de prevenção do suicídio e da autolesão voltadas para adolescentes em ambientes escolares? Os critérios de elegibilidade foram estudos originais publicados em português, inglês ou espanhol no período de 2017 a 2021, que apontassem estratégias em ambientes escolares voltadas para adolescentes na faixa etária de 10 a 19 anos ou para os profissionais que atuam no referido local. Os critérios de exclusão foram os estudos de caso e estudos de revisão de literatura.

O levantamento bibliográfico ocorreu no período de setembro a outubro de 2021, nas seguintes bases de dados online: Medical Literature Analysis and Retrievel System Online (MEDLINE), Literatura Latino-americana e do Caribe em Ciências da Saúde (LILACS), Índice Bibliográfico Español en Ciências de La Salud (IBECS), Base de Dados em Enfermagem (BDENF), Coleciona SUS e Index Psicologia- Periódicos pesquisadas via portal da Biblioteca Virtual em Saúde (BVS) e na base Education Resources Information Center ( ERIC).

A busca de artigos foi baseada na combinação dos seguintes descritores, disponíveis na lista de descritores em ciências da saúde da BVS (https://decs.bvsalud.org/): "adolescente", "suicídio", "ideação suicida", "tentativa de suicídio", "comportamento autodestrutivo", "automutilação", "autolesão", "serviço de saúde escolar", "escola" e "pessoal da educação" . Também foram utilizados os correspondentes na língua inglesa, presentes no Mesh terms: "adolescent”, "suicide”, "suicidal ideation", "suicide attempted", "self-injurious behavior", "self-mutilation", "self-harm", "school health services", "schools" e "educational personnel". A seguinte chave de busca foi utilizada nas bases pesquisadas: (adolescent) AND ("suicide" OR " 
suicidal ideation" OR "suicide attempted" OR "self-injurious behavior" OR "self- mutilation" OR "self- harm") AND ("school health services" OR "schools" OR "educational personnel").

A seleção de estudos foi realizada em duas fases: a primeira foi a leitura dos títulos e resumos por dois avaliadores independentes (AMPRE e MFAA) com auxílio do software Rayyan e a segunda a leitura do estudo na íntegra. Em caso de divergência entre os dois avaliadores, um terceiro autor (JELS) avaliou e, por consenso, foi tomada a decisão final sobre os estudos que seriam incluídos na revisão. Após a inclusão dos estudos foram extraídas as seguintes variáveis: base de dados, periódico, autor, título, ano, idioma, país, objetivo, população do estudo, idade estudada, desenho do estudo, tamanho da amostra, descrição da estratégia ou programa, instrumentos utilizados para avaliar a eficácia da intervenção ou programa, resultados alcançados e principais conclusões dos autores.

\section{Resultados e Discussão}

A presente pesquisa, inicialmente, obteve um total de 559 artigos, nas seguintes bases de dados online: MEDLINE, LILACS, IBECS, BDENF- Enfermagem, Coleciona SUS, e Index Psicologia Periódicos via portal da BVS e na base ERIC. Ao realizar o processo de exclusão dos artigos duplicados, esta quantidade passou a ser de 543 artigos. Posteriormente, foram analisados os artigos conforme os critérios de inclusão e exclusão, através das leituras dos títulos e resumos, os quais foram selecionados 24 artigos. Ressalta-se que 2 artigos foram excluídos nessa fase por não apresentarem acesso gratuito, resultando em 22 artigos para análise. Em seguida, houve a realização da leitura na íntegra dos artigos, resultando num total de 11 artigos que atenderam aos critérios de elegibilidade para compor esta revisão integrativa, conforme descrito na Figura 1. 
Figura 1 -Diagrama PRISMA referente às etapas de busca e seleção dos estudos da revisão integrativa.

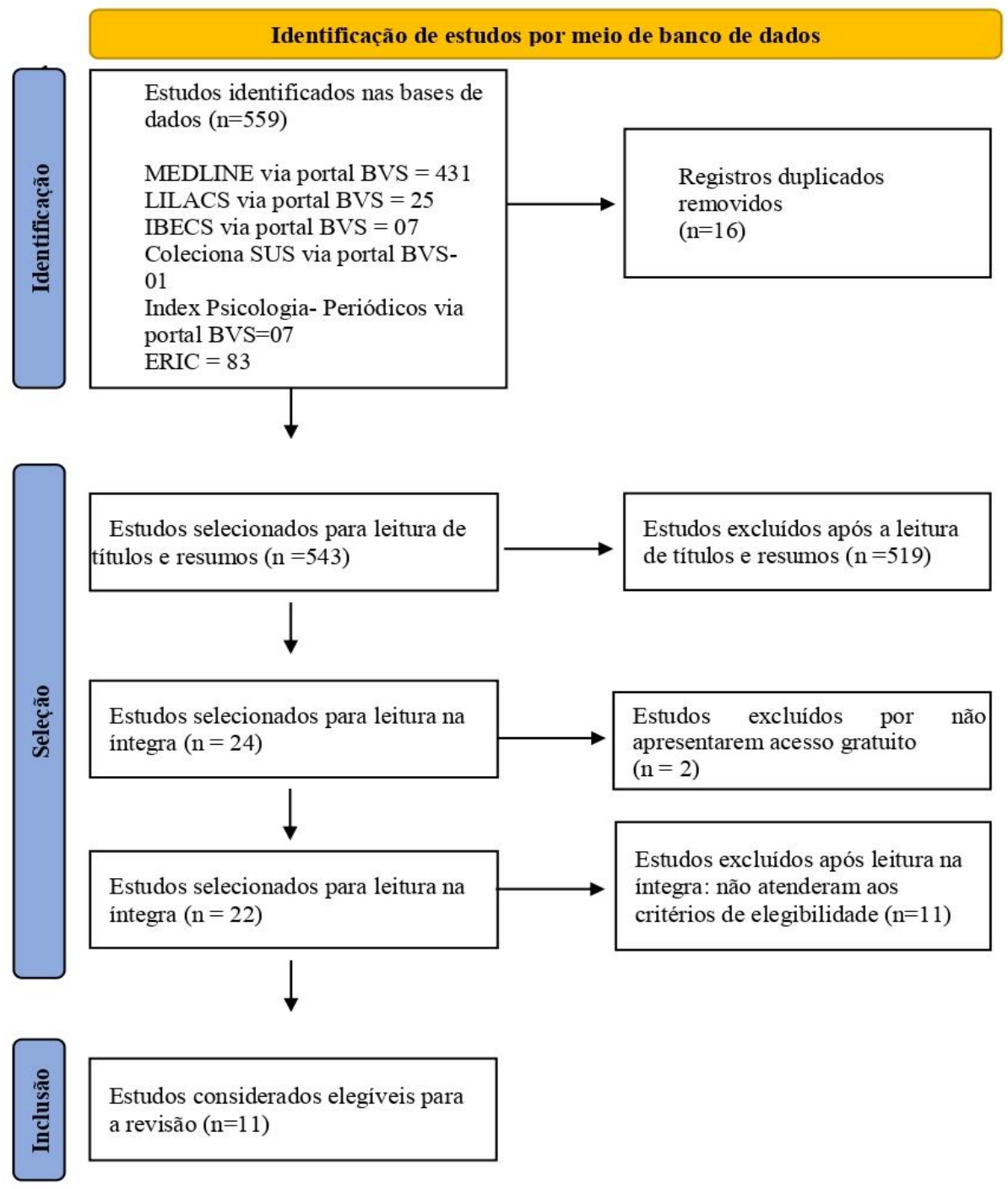

Fonte: Autores (2022)

\subsection{Caracterização dos artigos incluídos}

Os artigos incluídos nesta revisão foram publicados entre os anos de 2017 - 2021 e com predominância do idioma inglês. No que tange aos locais de estudo, estes desenvolveram-se em 8 países, sendo grande parte na Austrália (3 publicações) e nos Estados Unidos da América (2 publicações). Quanto a abordagem das publicações, 10 estudos estavam direcionados a temática do suicídio enquanto apenas 1 estudo abrangeu o suicídio e a autolesão.

No que concerne ao tópico intervenção realizada ou programa realizado, têm-se que 3 estudos explanaram sobre intervenções, sejam elas: a estratégia denominada Beyond The Wall com aplicação de questionário pré-intervenção e pós intervenção; a estratégia de currículo de alfabetização em saúde mental baseado em Harry Potter com a utilização da educação em saúde mental e Terapia Cognitivo Comportamental (TCC); a estratégia de intervenção Youth Aware of Mental Health (YAM) com uso da promoção da saúde mental na escola e prevenção primária do suicídio para adolescentes.

Em relação aos que abordaram sobre programa realizado, estes são divididos em 8 estudos: Programa de Prevenção do Suicídio Question Persuade Refer (QPR), que tem o objetivo de capacitar a comunidade local e escolar a identificar 
possíveis sinais de suicídio; Programa de Saúde Mental - Projeto KOICA - Yonsei, que tem a função de promover saúde mental no ambiente escolar; Programa Aussie Optismm, que tem como objetivo reduzir e prevenir os casos de ansiedade, depressão e comportamento suicida; Programa de Prevenção Escalonada Multimodal, que tem como função o uso da avaliação clínica, treinamento de Gatekeeper para redução de estigma e comportamento suicida; o SafeTALK, que tem como objetivo a realização de workshops para identificação de sinais de suicídio; Programa Silence is Deadly, direcionado para prevenção do suicídio e busca ativa precoce dos casos; Programa Jason Foundation (JF), tem a função de conscientizar sobre a prevenção do suicídio e interação com os jovens que podem estar em situação de risco; Programa On Peer Educators, com o objetivo de realizar escuta ativa e atenção plena às emoções. A síntese dos estudos incluídos para essa revisão integrativa estão no Quadro 1.

Quadro 1 - Síntese dos artigos incluídos na revisão.

\begin{tabular}{|c|c|c|c|}
\hline $\begin{array}{l}\text { Título do Artigo/Autores/Ano } \\
\text { Localidade }\end{array}$ & $\begin{array}{l}\text { Desenho do } \\
\text { Estudo/População }\end{array}$ & $\begin{array}{l}\text { Intervenção ou } \\
\text { Programa } \\
\text { Identificado no } \\
\text { Estudo }\end{array}$ & $\begin{array}{l}\text { Instrumentos para Análise da Intervenção ou } \\
\text { Programa }\end{array}$ \\
\hline $\begin{array}{l}\text { Beyond The Wall: Death } \\
\text { Education at Middle School as } \\
\text { Suicide Prevention } \\
\text { Testoni } \text { et al., } 2020 \\
\text { Itália }\end{array}$ & $\begin{array}{l}\text { Estudo Experimental } \\
\text { Adolescentes escolares de } 12 \\
\text { a14 anos }\end{array}$ & $\begin{array}{l}\text { Intervenção Beyond } \\
\text { The Wall }\end{array}$ & $\begin{array}{l}\text { Escala de Resiliência para Adolescentes (Escala } \\
\text { Likert), Questionário de Alexitima para Crianças } \\
\text { (AQC), Escala de Desespero para Crianças (HSC) } \\
\text { e Escala de Bem-Estar Infantil de Stirling } \\
\text { (SCWBS). }\end{array}$ \\
\hline $\begin{array}{l}\text { Culturally Informed Gatekeeper } \\
\text { Training For Youth Suicide } \\
\text { Prevention in Guyana: A Pilot } \\
\text { Examination. } \\
\text { Persaud } \text { et al., } 2019 \\
\text { Guiana }\end{array}$ & $\begin{array}{l}\text { Estudo Experimental } \\
\begin{array}{l}\text { Professores e funcionários } \\
\text { escolares }\end{array}\end{array}$ & $\begin{array}{lr}\text { Programa } & \text { de } \\
\text { Prevenção } & \text { do } \\
\text { Suicídio } & \text { Question } \\
\text { Persuade } & \text { Refer } \\
\text { (QPR) } & \end{array}$ & $\begin{array}{l}\text { Escala de Atitudes em Relação ao Suicídio } \\
\text { (ATTS), Perfil de Avaliação do Programa de } \\
\text { Prevenção de Suicídio (SPPRP) e entrevistas } \\
\text { semiestruturadas }\end{array}$ \\
\hline $\begin{array}{l}\text { Effectiveness of a School-Based } \\
\text { Mental Health Education } \\
\text { Program in an Impoverished } \\
\text { Urban Area of Peru } \\
\text { Kim } \text { et al., } 2020 \\
\text { Peru }\end{array}$ & $\begin{array}{l}\text { Estudo Experimental } \\
\text { Adolescentes escolares da } 1^{\mathrm{a}} \\
\text { a } 5^{\mathrm{a}} \text { série do ensino médio }\end{array}$ & $\begin{array}{l}\text { Programa de Saúde } \\
\text { Mental- Projeto } \\
\text { KOICA - Yonsei }\end{array}$ & $\begin{array}{llll}\text { Questionário } & \text { Global } & \text { School-Based } & \text { Student } \\
\text { Health Survey } & & & \end{array}$ \\
\hline $\begin{array}{l}\text { Efficacy of the Aussie } \\
\text { Optimism Program: Promoting } \\
\text { Pro-social Behavior and } \\
\text { Preventing Suicidality in } \\
\text { Primary School Students. } \\
\text { A Randomised-Controlled Trial } \\
\text { Roberts } \text { et al., } 2017 \\
\text { Austrália }\end{array}$ & $\begin{array}{l}\text { Ensaio } \\
\text { Controlado } \\
\begin{array}{l}\text { Diretores, professores e } \\
\text { alunos }\end{array}\end{array}$ & $\begin{array}{l}\text { Programa Aussie } \\
\text { Optismm }\end{array}$ & $\begin{array}{l}\text { Questionário de Pontos Fortes e Dificuldades } \\
\text { (SDQ-S e SDQ-P) e Diagnóstico para Crianças e } \\
\text { Adolescentes IV (DICA-IV). }\end{array}$ \\
\hline $\begin{array}{l}\text { Evaluation of a Multimodal } \\
\text { School-Based } \\
\text { and Suicide Prevention Program } \\
\text { Among Dutch adolescents: } \\
\text { Design of a Cluster- } \\
\text { Randomized Controlled Trial } \\
\text { Gijzen } \text { et al., } 2018 \\
\text { Holanda }\end{array}$ & $\begin{array}{l}\text { Ensaio Clínico Randomizado } \\
\text { Controlado } \\
\text { Adolescentes escolares de } 11 \\
\text { a } 15 \text { anos }\end{array}$ & $\begin{array}{l}\text { Programa de } \\
\text { prevenção } \\
\text { escalonada } \\
\text { multimodal baseado } \\
\text { em escola para } \\
\text { depressão e } \\
\text { comportamentos } \\
\text { suicidas em } \\
\text { adolescentes }\end{array}$ & $\begin{array}{l}\text { Questionário de Avaliação de Suicídio e } \\
\text { Autolesão (VOZZ), Inventário de Depressão } \\
\text { Infantil } 2 \text { (CDI-2) e Persuasão e Consulte (QPR). }\end{array}$ \\
\hline $\begin{array}{l}\text { Program Evaluation and } \\
\text { Decision Analytic Modelling of } \\
\text { Universal Suicide Prevention } \\
\text { Training (SafeTALK) in } \\
\text { Secondary Schools } \\
\text { Kinchin } \text { et al., } 2020 \\
\text { Austrália }\end{array}$ & $\begin{array}{l}\text { Estudo Experimental } \\
\text { Adolescentes de } 15 \text { a } 19 \text { anos }\end{array}$ & $\begin{array}{l}\text { Programa } \\
\text { SafeTALK }\end{array}$ & Modelo Kirkpatrick \\
\hline $\begin{array}{l}\text { Silence is Deadly: A Controlled } \\
\text { Trial of a Public Health }\end{array}$ & $\begin{array}{l}\text { Ensaio Clínico Controlado de } \\
\text { dois braços com uma }\end{array}$ & $\begin{array}{l}\text { Programa Silence is } \\
\text { Deadly }\end{array}$ & $\begin{array}{l}\text { Questionário de Busca de Ajuda Geral, Versão } \\
\text { Adaptada do Questionário de Busca de Ajuda }\end{array}$ \\
\hline
\end{tabular}




\begin{tabular}{|c|c|c|c|}
\hline $\begin{array}{l}\text { Intervention to Promote Help- } \\
\text { Seeking in adolescent males } \\
\text { Calear et al., } 2021 \\
\text { Austrália }\end{array}$ & $\begin{array}{l}\text { intervenção ativa e grupo de } \\
\text { controle de lista de espera } \\
\text { Adolescentes do sexo } \\
\text { masculino de } 16 \text { a } 18 \text { anos }\end{array}$ & & $\begin{array}{l}\text { Real, Atitudes em Relação à Procura de Ajuda } \\
\text { Profissional Psicológica: Formulário resumido } \\
\text { (SSOSH), Escala de Atitudes para Buscar Apoio } \\
\text { de Adultos de Confiança, Escala Autoestigma de } \\
\text { Busca de Ajuda, Distress Questionnaire-5, Um } \\
\text { item do Inquérito ao comportamento de Risco de } \\
\text { Jovens, Subescala de Emocionalidade Restritiva } \\
\text { da Escala de Conflito de Papel de Gênero e duas } \\
\text { perguntas adaptadas da literatura avaliando o } \\
\text { programa de primeiros socorros em saúde mental. }\end{array}$ \\
\hline $\begin{array}{l}\text { The Impact of a Harry Potter- } \\
\text { Based Cognitive-Behavioral } \\
\text { Therapy Skills Curriculum on } \\
\text { Suicidality and Well-being in } \\
\text { Middle Schoolers: } \\
\text { A Randomized Controlled Trial. } \\
\text { Klin-Conforti et al., } 2021 \\
\text { Canadá }\end{array}$ & $\begin{array}{l}\text { Ensaio Clínico Randomizado } \\
\text { Adolescentes escolares de } 11 \text { - } \\
14 \text { anos }\end{array}$ & 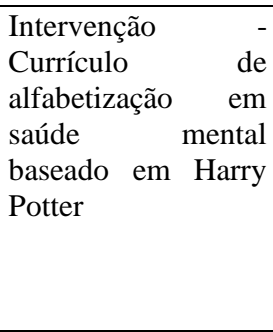 & $\begin{array}{l}\text { Inventário de Problemas de Vida (LPI) e Escala } \\
\text { Revisada de Ansiedade e Depressão Infantil } \\
\text { (RCADS) }\end{array}$ \\
\hline $\begin{array}{l}\text { The Role of Youth Trainee- } \\
\text { Trainer Alliance r and } \\
\text { Involvement in School-Based } \\
\text { Prevention: A Moderated- } \\
\text { Mediation Model of } \\
\text { Student Gatekeeper Suicide } \\
\text { Prevention Training } \\
\text { Totura et al., } 2019 \\
\text { EUA }\end{array}$ & $\begin{array}{l}\text { Estudo Experimental } \\
\text { Adolescentes escolares do } \\
\text { ensino médio }\end{array}$ & $\begin{array}{lr}\text { Programa } & \text { Jason } \\
\text { Foundation (JF) "A } & \text { Promise } \\
\text { Tomorrow" } & \\
\text { Gatekeeper } & \\
\text { prevention } & \end{array}$ & $\begin{array}{lll}\text { Questionário de } & \text { Implementação } & \text { e } \\
\text { Comportamento Planejado (PBIQ) } & \end{array}$ \\
\hline $\begin{array}{l}\text { The Youth Aware of Mental } \\
\text { Health Intervention: Impact on } \\
\text { Help Seeking, Mental Health } \\
\text { Knowledge, and Stigma in U.S. } \\
\text { Adolescents } \\
\text { Lindow et al., } 2020 \\
\text { EUA }\end{array}$ & $\begin{array}{l}\text { Estudo Experimental com } \\
\text { desenho pré-teste e pós-teste } \\
\text { não controlado } \\
\text { Adolescentes escolares, com } \\
\text { média de idade de } 14,5 \text { anos }\end{array}$ & $\begin{array}{ll}\text { Intervenção } & \text { Youth } \\
\text { Aware of } & \\
\text { Mental } & \text { Health } \\
\text { (YAM) } & \end{array}$ & $\begin{array}{l}\text { Questionário de Busca de Ajuda Geral (GHSQ) } \\
\text { e Escala de Comportamento Relatado e } \\
\text { Pretendido }\end{array}$ \\
\hline $\begin{array}{l}\text { What Is in It for Them? } \\
\text { Understanding the Impact of a } \\
\text { 'Support, Appreciate, Listen } \\
\text { Team' (SALT) - Based Suicide } \\
\text { Prevention Peer Education } \\
\text { Program on Peer Educators } \\
\text { Zachariah, et al., } 2018 \\
\text { India }\end{array}$ & $\begin{array}{l}\text { Estudo Qualitativo } \\
\text { Adolescentes escolares de } 12 \\
\text { a } 15 \text { anos }\end{array}$ & $\begin{array}{l}\text { Programa On Peer } \\
\text { Educators }\end{array}$ & $\begin{array}{l}\text { Métodos mistos de coleta de dados qualitativos, } \\
\text { incluindo grupos focais, entrevistas } \\
\text { semiestruturadas e questionários abertos }\end{array}$ \\
\hline
\end{tabular}

Fonte: Elaborado pelos autores (2022).

\subsection{Intervenções}

\section{Intervenção Beyond The Wall}

A primeira intervenção, definida como Beyond The Wall, inserida no estudo dos autores Testoni et al (2020), tem como objetivo fornecer informações sobre a morte que sejam apoiadas por uma linguagem adequada para auxiliar na compreensão emocional, produzir reflexões sobre o sentido da vida e apoiar o compartilhamento de experiências com amigos e colegas de classe.

Nos encontros, houve a realização de exibição de filmes, psicodrama e photovoice, com o objetivo de centrar as expectativas para o futuro. O estudo foi realizado com uma população de adolescentes entre 12 e 14 anos e avaliou fatores relacionados com sentimentos e competências infanto-juvenis, como resiliência, bem-estar e alexitimia, utilizando questionários pré intervenção e pós intervenção. Durante a aplicação desta intervenção observou-se que no grupo experimental foram encontrados níveis mais elevados de incompreensão das emoções. Nos grupos experimental e controle, houveram 
constatações de correlações positivas e negativas para os seguintes pontos: alexitimia e desesperança e resiliência e bem-estar na infância. Para o grupo experimental, dois fatores emocionais apresentaram declínio entre o pré e pós teste: a Difficulty Describing Your Feelings (DDF) e o Externally Oriented Thinking (EOT), isto é, como a criança/adolescente está expondo seus sentimentos. Todavia, três fatores emocionais obtiveram pontuações menores em ambos os grupos, mesmo após a intervenção: Dificuldade em Identificar Sentimentos, Recursos Sociais e Competência Social. Os autores concluíram que embora alguns destes fatores tenham apresentado alguma diferença durante a execução do treinamento, sejam elas positivas ou negativas, estas não foram significativas e não obtiveram grandes impactos quando em conjunto, apenas individualmente (Testoni et al, 2020).

Silva et al (2017) discutiram a importância da realização da promoção da saúde mental através da arte e da cultura, nas quais, estas têm o papel de empoderar os adolescentes à nível individual e coletivo, diferindo, portanto, do trabalho de Testoni et al (2020), que utilizam da temática da morte para elaborar a intervenção, que apresenta um pequeno grau de eficácia apenas no modo individual.

\section{Intervenção Currículo de alfabetização em Saúde Mental baseado em Harry Potter}

A segunda intervenção refere-se a um estudo baseado no currículo de alfabetização em saúde mental com base em Harry Potter, dos autores Klim-Conforti et al (2021). Esta estratégia foi realizada com uma população de adolescentes de 11 a 14 anos, na qual transmitiu habilidades de TCC, sobre suicídio e bem-estar no referido público. A estratégia ocorreu durante as aulas de inglês dos estudantes do ensino médio, onde os pontos abordados apresentaram um enfoque no incentivo à flexibilidade cognitiva, a resiliência e controle, por meio do uso dos denominados "destruidores do estresse". Para a realização desta intervenção, os pesquisadores forneceram tanto para os alunos quanto para os professores, um manual de atividades. Diante disso, foram encontrados os seguintes resultados: ambos os grupos, intervenção e controle, obtiveram significância estatística, no entanto, o primeiro grupo adquiriu respostas mais positivas.

Nasution, Keliat \& Wardani (2019) também utilizaram a TCC em seu estudo e verificaram que esta, juntamente com outras abordagens mostraram-se bastante eficazes na diminuição da ideação suicida. A utilização do romance Harry Potter, obra literária bastante contemplada entre os adolescentes, representa uma estratégia de intervenção que busca tanto compreender esta fase da vida e os desafios atribuídos a ela, quanto a singularidade de cada participante. Tal ação pode ser vista no estudo desenvolvido por Neves, Pereira \& Pereira (2020), que ressalta a importância das ações de prevenção observarem as especificidades e afinidades comuns da adolescência.

\section{Intervenção baseada no Youth Aware of Mental Health (YAM)}

A terceira estratégia de intervenção aborda uma pesquisa baseada no YAM, que é definida como um método de conscientização da saúde mental e da prevenção do suicídio apropriado para a fase da adolescência. O YAM aborda em sua metodologia o desenvolvimento de habilidades, conhecimentos e consciência emocional, fatores essenciais para o enfrentamento dos pensamentos e comportamentos suicidas. Lindow et al (2020) em seu estudo objetivaram determinar a viabilidade e aceitabilidade do YAM adaptado para jovens dos EUA com a população de adolescentes na faixa etária média de 14,5 anos.

Para realização desta intervenção, foi utilizado um modelo universal de promoção da saúde mental sendo a escola o local escolhido para aplicá-lo. A intervenção é produzida num tempo de 50 minutos com cinco sessões, nas quais, são trabalhados os seguintes pontos: dramatização, palestras sobre saúde mental, livreto informativo e uso de pôsteres. Ao executar esta estratégia, os autores encontraram como resultado quatro temáticas que foram divididas em categorias. A primeira está relacionada ao comportamento de busca de ajuda, onde os alunos apresentaram uma taxa de crescimento em relação a 
conversar com os amigos sobre questões ligadas à saúde mental, assim como, dialogar com os professores e outros profissionais do ambiente escolar sobre depressão e pensamentos suicidas. Esta resposta mostrou-se positiva após 3 meses de intervenção. A segunda temática refere-se ao comportamento de intenção de ajuda, em que não foram detectadas alterações ao longo do tempo, quando os adolescentes foram questionados sobre buscas de ajuda nas problemáticas direcionadas à saúde mental. A terceira temática relata a procura de ajuda em matéria de saúde mental e conhecimento de recursos, no qual, os adolescentes, após a intervenção, apresentaram um aumento no nível de conhecimento sobre este tema. A última temática descreve sobre o estigma relacionado à saúde mental, que expõe acerca da melhoria na redução do estigma, 3 meses após a intervenção (Lindow et al, 2020).

Os autores concluíram que esta estratégia de intervenção baseada no YAM é eficaz e protege contra o suicídio, assim como, fatores que podem levar a este ato, como por exemplo o estigma. Esta afirmação também pode ser encontrada no estudo feito por Kahn et al (2020) que evidenciou o YAM como uma intervenção capaz de reduzir a ideação suicida e que o processo de socialização, próprio da estratégia, é um ponto forte da sua eficácia. Porém, estes autores destacam que mais estudos precisam ser elaborados para discutir como o YAM atua nos adolescentes a partir da sua combinação com outras estratégias.

\subsection{Programas}

\section{Programa de Prevenção do Suicídio Question Persuade Refer (QPR)}

O Programa é caracterizado como um treinamento de Gatekeeper (guardiões da vida) que ensina professores, pais e outros trabalhadores comunitários como reconhecer os sinais de suicídio, realizar intervenções de saúde mental e encaminhar pessoas em risco de morrer por suicídio para um profissional de saúde mental. A sigla QPR refere-se à técnica de intervenção central do treinamento, a saber: como fazer a pergunta do suicídio com exemplos de fraseologia e resultados previstos; como persuadir uma pessoa suicida a aceitar ajuda (habilidades de escuta ativa, foco no(s) problema(s), pedidos de ação para salvar vidas); e, como encaminhar uma pessoa suicida para recursos locais/nacionais (acompanhados de referência, nomes, números, endereços). O treinamento é ministrado por um instrutor certificado de QPR e inclui componentes didáticos baseados em palestras, discussão e dramatização (Persaud et al., 2019).

Persaud et al (2019) investigaram a eficácia, aceitabilidade e viabilidade do referido programa, os autores realizaram três momentos distintos de análise, no primeiro momento foi aplicado pré e pós- teste sobre a prevenção do suicídio e escala de Atitudes em Relação ao Suicídio (ATTS) resultando em efeito positivo, no segundo momento houve aplicação do teste de avaliação do programa no qual destaca-se que as pontuações de aceitabilidade foram altas e no terceiro momento examinada a viabilidade deste. Portanto, os autores concluíram quanto a importância do treinamento Gatekeeper de prevenção de suicídio para professores e outros funcionários da escola que convivem diariamente com os adolescentes em risco de suicídio, além disso demonstraram a eficácia do programa para adolescentes de baixa renda.

Corroborando com o exposto, Terpstra et al (2018) realizaram um estudo observacional com profissionais submetidos ao treinamento de Gatekeeper, concluindo que, houve um aumento significativo do conhecimento e das habilidades de prevenção do suicídio dos envolvidos no estudo. Ademais, Brito et al (2020) analisaram os conhecimentos sobre estratégia de prevenção de suicídio adotadas por professores do ensino fundamental e revelaram que há necessidade de ações voltadas para a capacitação desses profissionais, enfatizando a prevenção e identificação dos adolescentes em risco, com manejo adequado e encaminhamento compartilhado aos serviços de saúde. 


\section{Programa de Saúde Mental- Projeto KOICA - Yonsei}

O Programa de Saúde Mental - Projeto KOICA - Yonsei é definido como um programa de promoção da saúde escolar, focado na saúde mental, voltado para os adolescentes. Para a execução deste, faz-se necessário que seja avaliado a cada ano, seu principal objetivo é reduzir as taxas de tentativa de suicídio (Kim et al., 2020).

A fim de pesquisar sobre a eficácia deste Programa, Kim et al (2020), realizaram em seu estudo as seguintes avaliações: questionários pré-intervenção e pós intervenção. Este instrumento foi autoaplicável e elaborado através da Pesquisa Global de Saúde do Aluno com Base na Escola de 2010 e os Módulos do Questionário da Pesquisa Global de Saúde do Aluno com Base na Organização Mundial da Saúde. O questionário Global School-Based Student Health Survey tem como objetivo avaliar tanto os fatores de risco quanto os de proteção relacionados ao comportamento dos adolescentes. Diante disso, foram encontrados alguns resultados, sejam eles: para a análise estatística os grupos experimental e controle mostraram-se homogêneos. O estudo avaliou algumas temáticas e suas correlações com o programa, como, fatores de predisposição, reforço e habilitação; efeito do programa em fatores psicológicos; efeito do programa nas tentativas de suicídio. Na temática de fatores de predisposição, reforço e habilitação, a variável com maior diferença estatística foi o consumo de tabaco, no que se refere ao tema efeito do programa em fatores psicológicos, a variável que apresentou maior diferença estatística foi autoavaliação de saúde e na temática de efeito do programa nas tentativas de suicídio, a variável com maior diferença estatística foi felicidade subjetiva. Os autores então concluíram que a implantação do Programa de Saúde Mental melhorou a intenção de mudança do comportamento do adolescente nos fatores relacionados à predisposição, reforço, habilitação, comportamentais, ambientais e psicológicos.

Cervantes e Contreras (2019) conduziram uma pesquisa sobre a relação do consumo de tabaco e álcool por adolescentes e concluíram que o crescimento da ingestão de tabaco aumenta a taxa de prevalência de suicídio em duas vezes, já o álcool, tem uma elevação de três vezes mais confirmando, portanto, os resultados significativos apontados por Kim et al (2020).

Em pesquisa realizada por Severian et al (2021) com estudantes de graduação em enfermagem sobre a eficácia da implementação de uma intervenção psicoeducacional, esta não demonstrou níveis positivos de melhora da ansiedade e depressão após a aplicação da estratégia, divergindo da proposta trabalhada por Kim et al (2020) que relata melhoria nos fatores de comportamento dos adolescentes.

\section{Programa Aussie Optismm (AOP)}

Este Programa é estruturado através de uma ação realizada por meio de uma estratégia universal de promoção da saúde mental, que objetiva reduzir e prevenir a ansiedade, depressão e comportamento suicida em adolescentes. O conteúdo contempla habilidades sociais, emocionais e cognitivas, tendo como principais aspectos: identificação de sentimentos, tomada de decisão, comunicação, assertividade e negociação, habilidades de enfrentamento e redes de apoio social, conectando pensamentos e sentimentos, estilos de pensamento, desafiando estilos de pensamento negativo e preparando-se para a adolescência. Os autores utilizaram na análise do estudo os instrumentos Strenghs and Difficulties Questionaire (SDQ-S e SDQ-P) sendo o primeiro direcionado para a avaliação de saúde mental infanto-juvenil e o segundo para os pais; Diagnóstico para Crianças e Adolescentes IV (DICA IV) que avalia a depressão e ansiedade em momentos anteriores e no presente (Roberts et al.,2017).

Outrossim, a importância da utilização deste Programa no ambiente escolar, com a participação de pais e alunos, confirma o que foi implantado no estudo desenvolvido por Guedes, Mendes \& Messias (2019), que relatam sobre a escola, como local de acolhimento e vínculo para com os adolescentes, sendo assim um espaço apropriado para a realização de educação em saúde mental. Neste sentido, Lima et al (2019) sugere em sua pesquisa que introduzir políticas e programas de 
saúde baseadas na escola é algo efetivo, uma vez que esta utiliza dos mecanismos de diálogo, redes de apoio e habilidades sociais para implantar ações e estratégias de prevenção de agravos para os adolescentes e suas famílias.

\section{Programa de Prevenção Escalonada Multimodal Baseado em Escola para Depressão e Comportamentos Suicidas em} Adolescentes

Este Programa é caracterizado como uma prevenção que utiliza a escola para desenvolver ações direcionadas à redução da depressão e dos comportamentos suicidas. É definido como uma triagem a partir do uso da avaliação clínica ou do encaminhamento para algum serviço que realiza atendimento de saúde; condução de treinamento para Gatekeeper para mentores; prevenção para diminuir o estigma; identificar adolescentes com aparente fator de risco para comportamentos suicidas e de autolesão (Gijzen et al., 2018)

Na pesquisa de Gijzen et al (2018), a população estudada contempla adolescentes na faixa etária dos 11 aos 15 anos de uma escola do ensino médio. Para avaliar a eficácia do Programa, serão utilizados três instrumentos, sejam eles: Questionário de avaliação de suicídio e autolesão (VOZZ), que tem a função de avaliar o risco de suicídio e autolesão, o Inventário de Depressão Infantil 2 (CDI-2) que tem em sua composição 28 itens para avaliar sintomas depressivos e Persuasão e Consulte (QPR), onde os responsáveis pelos adolescentes serão denominados de guardiões, no qual haverá um treinamento para reconhecer e compreender os comportamentos suicidas. Os resultados encontrados relatam que é importante análises de moderação através de variáveis como gênero, descendência étnica/histórico cultural, nível de perfeccionismo de base e nível de depressão de CDI-2. Faz-se necessário o desenvolvimento de uma análise mais abrangente para compreender o número de casos de suicídio no grupo intervenção e no controle. Os autores ainda ressaltam que se houver diminuição entre os casos de comportamentos suicidas, autolesivos e sintomas depressivos nos adolescentes, este Programa deve ser implantado nas escolas em larga escala.

Mansour et al (2020) realizaram um estudo com o objetivo de introduzir um projeto baseado no questionário relacionado à saúde mental para monitorar os casos de depressão entre os adolescentes atendidos no Centro de Saúde Federal Qualificado. Os autores inferiram que, após a instauração do projeto, houve um aumento exponencial no que tange tanto ao número de triagens desenvolvidas quanto ao encaminhamento de adolescentes para os serviços de saúde mental. Os autores ainda ressaltam que a Atenção Primária à Saúde pode representar um importante elo para a amplificação deste rastreamento. Portanto, este projeto ratifica a importância da implantação da prevenção escalonada multimodal, no tocante a necessidade da avaliação clínica e do encaminhamento para serviços especializados, como fator importante para a redução dos casos de suicídio.

\section{Programa SafeTALK}

O Programa SafeTALK foi desenvolvido a partir de um workshop com duração de 3 horas sendo realizado pela Living Works Education no ano de 2006. Ao longo de sua execução foram ofertadas apresentações, vídeos, diálogos sobre a temática de suicídio e questionamentos. As discussões abrangem quatro tópicos: saber identificar os possíveis sinais de suicídio; retirar a visão de evitar falar sobre tal temática; utilizar a sigla FALAR (diga, pergunte, ouça e mantenha a segurança) para intervir; realizar vínculo entre a pessoa que necessita de ajuda com o serviço de saúde e sociedade como um todo. O Programa foi realizado através de treinamento que incluiu apresentações, vídeos e discussões. Foi disponibilizado um cartão com a presença de etapas para alerta do suicídio. $\mathrm{O}$ workshop foi facilitado por um instrutor de Treinamento de Habilidades de Intervenção Aplicada ao Suicídio e SafeTALK (Kinchin et al., 2020).

Para testar a eficácia do SafeTALK, Kinchin et al (2020) utilizaram como instrumento o Modelo Kirkpatrick, que consiste em avaliar a intervenção antes e após o Programa. Esta avaliação foi realizada por meio de quatro níveis, quais sejam: 
reação (Nível I), aprendizagem (Nível II), comportamento (Nível III) e resultados (Nível IV), com uma população de adolescentes de 15 a 19 anos, no Mercy College. Com isso, os resultados obtidos pelos autores demonstram que houve uma análise pré e pós acompanhamento, com a presença de uma significativa perda de participantes ao longo da sua execução pelo fato de os alunos estarem no final do período letivo. Destaca-se que houve a avaliação da eficácia, em que as variáveis conhecimento, confiança, disposição e busca de ajuda, pontuaram positivamente antes e após a implementação do programa. Verificou-se que durante o pré e pós treinamento o número de sinais de alerta de suicídio aumentou e nas nuvens de palavras, as mais citadas foram depressão, antisocial, autoagressão, tristeza e ansiedade. No que tange a busca de ajuda, as pessoas às quais os alunos recorreriam seriam amigos, pais, profissionais de saúde mental e linhas de apoio de telefone. Quanto às barreiras para procura de ajuda, os motivos mais frequentes estão relacionados com vergonha, timidez, julgamento do outro e preocupação sobre a condução do tratamento. No que se refere a ajudar o outro aluno nos momentos de angústia, grande parte dos adolescentes mencionaram que oferecem esse tipo de ação. Ressalta-se que foi unânime a aprovação do Programa pelos participantes. Ao concluírem, os autores refletiram que a aplicação do Programa deve ser feita em grupos de até 15 alunos e que avaliações futuras do SafeTALK são necessárias para observar como se comportam com um grupo controle.

Anteriormente ao estudo citado, Stewart et al. (2018) buscaram discutir a eficácia do SafeTALK. Os resultados encontrados diferem da pesquisa de Kinchin et al (2020) no que se refere ao aumento da autoeficácia e da aprovação da implantação do Programa pelos participantes do sexo masculino. Em 2020, não houve avaliação do Programa considerando apenas a variável sexo. Todavia, o estudo de 2018 corrobora com o desenvolvido em 2020, que relata sobre a importância da realização de aspectos que aumentem a eficácia do SafeTALK.

Uma das temáticas abordadas no estudo de Kinchin et al (2020) foram as palavras que mais representaram os sinais associados aos comportamentos suicidas. O trabalho de Chang (2017) relata que a desesperança, a tristeza e a falta de visão do futuro são os termos mais utilizados pelos adolescentes e estão diretamente correlacionadas quando estes relatam os comportamentos suicidas.

\section{Programa Silence is Deadly}

O Programa é uma intervenção de saúde pública padronizada, desenvolvida para prevenir o suicídio por meio da promoção da busca precoce de ajuda para problemas emocionais. É ministrado em escolas secundárias e aborda diretamente alguns dos fatores-chave (conhecimento, atitudes e normas) que supostamente causam impacto no comportamento de procura de ajuda para problemas emocionais e suicídio, é operacionalizado com uma apresentação de 45 a 60 minutos, site de apoio, vídeos e apostilas informativas. É destinado para adolescentes do sexo masculino e emprega um desenho psicoeducacional no qual estatísticas importantes sobre saúde mental, suicídio e busca de ajuda são apresentadas e as maneiras pelas quais a masculinidade pode impedir a busca de ajuda precoce e eficaz. Embora o programa enfatize a busca e o fornecimento de suporte de pares, ele também reconhece a importância de buscar ajuda de fontes adultas, como pais, professores e profissionais de saúde (Calear et al., 2021).

Os autores testaram a eficácia do referido programa em adolescentes do sexo masculino, na faixa etária de 16 a 18 anos, na pesquisa utilizaram intrumentais com questões quantitativas, demográficas e qualitativas nos grupos de intervenção e controle, e concluíram que, as intenções de buscar ajuda de um amigo não diferiram entre os grupos de participantes nos momentos pré e pós-intervenção. No entanto, no acompanhamento, as intenções de busca de ajuda do grupo de intervenção aumentaram significativamente mais do que o grupo de controle. É importante ressaltar que o estudo demonstrou que o programa Silence is Deadly é valorizado por alunos e funcionários por sua relevância e que intervenções de saúde pública de baixa intensidade para suicídio têm o potencial de melhorar a procura de ajuda pelos adolescentes (Calear et al., 2021). 
Corroborando com a discussão Rasmussen et al (2018) referem que os homens jovens constituem um dos grupos de maior risco de suicídio na maioria dos países, os autores mencionaram como os significados ligados à masculinidade podem ser evocados quando um jovem tira sua vida. Neste sentido, é preciso compreender o papel de gênero na formação da identidade dos sujeitos para propor programas de prevenção do suicídio eficazes.

\section{Programa Jason Foundation (JF) “A Promise for Tomorrow” Gatekeeper Prevention}

O programa JF "A Promise for Tomorrow" Gatekeeper Prevention busca promover a conscientização sobre o problema do suicídio de jovens, fornece aos alunos estagiários conhecimento e recursos para interagir com adolescentes em situação de risco e incentiva comportamentos de encaminhamento. As sessões de treinamento Gatekeeper (guardiões da vida) foram conduzidas por seis professores de educação em saúde em duas escolas públicas urbanas de ensino médio e o conteúdo do treinamento foi ministrado em três aulas utilizando uma variedade de métodos de ensino, tais como: discussões entre alunos sobre sinais de alerta do comportamento suicida, trabalho em grupos com exercícios interativos e dramatizações baseadas em habilidades (Totura et al., 2019).

Os autores do estudo avaliaram as intençães dos alunos em utilizar as habilidades aprendidas após a participação no referido programa e utilizaram como instrumento o Questionário de Implementação e Comportamento Planejado (PBIQ) que incluiu itens como conhecimento de prevenção de suicídio de jovens, atitudes sobre prevenção de suicídio, intenções de se envolver em comportamentos de referência, aliança com o formador, percepção da participação dos colegas dentre outros. Portanto, comprovaram que quanto maior a aliança entre o educador e o adolescente e seu envolvimento com o programa, maiores serão as intenções em utilizar os conhecimentos e habilidades adquiridos após a participação no treinamento (Totura $e t$ al., 2019).

Mo et al (2018) realizaram a primeira revisão sistemática sobre a eficácia do treinamento Gatekeeper prevention em ambientes escolares, e concluíram que, o referido treinamento tem potencial de mudar o conhecimento e as habilidades dos participantes na prevenção do suicídio, entretanto são necessários estudos de melhor qualidade para determinar a eficácia na mudança de atitudes dos envolvidos no programa.

\section{Programa On Peer Educators}

Os Programas On Peer Educators (educação de pares) foram implementados em todo o mundo desde a década de 1960, sendo executados de diversas formas e para uma variedade de objetivos, e particularmente nas últimas décadas utilizados para abordar a saúde mental infanto-juvenil em idade escolar. Neste estudo, a filosofia básica que sustenta os mecanismos de funcionamento do Programa On Peer Educators é a capacidade humana de resposta, considerando que uma comunidade escolar pode enfrentar coletivamente os desafios, sobretudo quando os alunos recebem treinamentos e incentivos precisos podendo induzir uma mudança positiva na vida de seus colegas (Zachariah et al., 2018).

Neste sentido, o referido programa está focado na criação de uma rede eficaz de voluntários que estão engajados com seu ambiente direto, principalmente com seus colegas de escola. As etapas do programa são: (1) recrutamento de voluntários; (2) treinamento; e (3) orientação contínua e reflexão sobre os objetivos de trabalho como voluntários. Este processo é orientado por psicólogos profissionais e voluntários adultos treinados, ressaltando que ao longo do período letivo são previstas reuniões semanais na escola com objetivo de aprender, compartilhar abertamente e apoiar uns aos outros (Zachariah et al., 2018)

Zachariah et al (2018) estudaram as mudanças observadas em alunos que estiveram envolvidos em um programa de educação de pares baseado em atenção plena e escuta ativa para abordar o comportamento suicida, os adolescentes envolvidos no programa estavam na faixa etária de 12 a 15 anos. Os autores utilizaram métodos mistos de coleta de dados qualitativos, incluindo grupos focais, entrevistas semiestruturadas e questionários abertos, e concluindo que: houve um aumento da 
capacidade dos alunos de reconhecer o sofrimento dos outros; de chegar às pessoas em perigo; de usar habilidades de escuta e abordagens de não julgamento; e conduzir as atividades em equipe.

Eisenstein et al (2019) analisaram a eficácia de um projeto de educação de pares implementado para alunos do ensino médio. O projeto objetivava desenvolver as habilidades e os conhecimentos necessários para proteger a saúde mental dos adolescentes e de seus pares. Logo, os resultados indicaram que a participação no projeto está associada a uma melhoria significativa nas habilidades-chave dos alunos e estes recomendariam a participação no programa para outros adolescentes.

\section{Conclusão}

As intervenções encontradas nesta revisão de literatura abordaram questões de saúde mental através do fortalecimento das habilidades socioemocionais, nos quais apresentaram como principais pontos: a prevenção do suicídio com foco no saber lhe dar com as emoções negativas e valorização da vida; alfabetização em saúde mental enfatizando a flexibilidade cognitiva, resiliência e controle para prevenção dos comportamentos suicidas; desenvolvimento de habilidades, conhecimentos e consciência emocional, direcionados para o enfrentamento dos pensamentos e comportamentos de risco.

Enquanto as estratégias utilizadas nos programas estudados verificaram-se que houve predominância de treinamento para reconhecimento dos sinais do comportamento suicida e encaminhamento aos profissionais da saúde mental, principalmente o Gatekeeper (guardiões da vida). Observou-se que os ambientes escolares são espaços potenciais para intervenções educativas em saúde mental com ênfase na prevenção dos comportamentos suicidas e de autolesão, uma vez que a escola faz parte da rotina diária do adolescente e pode ser um local de construção de vínculos entre profissionais de educação, família e alunos.

Os estudos que compuseram a revisão em sua maioria demonstraram eficácia quanto aos resultados alcançados, utilizando-se de instrumentos validados para análise das intervenções e programas. No entanto, alguns estudos apresentaram algumas lacunas, sendo necessária a realização de novas pesquisas para melhor avaliar os programas e estratégias. Neste sentido, foram verificados que a intervenção Currículo de alfabetização em Saúde Mental baseado em Harry Potter e os Programas QPR, On Peer Educators e JF foram eficazes, entretanto precisam ser avaliados a longo prazo. Quanto à intervenção baseada no YAM, nos Programas de Saúde Mental- Projeto KOICA - Yonsei, Aussie Optismm (AOP) e na Prevenção Escalonada Multimodal foram evidenciados que estes também são eficazes, contudo precisam ser analisados numa escala populacional maior e ampliados para outros ambientes escolares. Em relação à intervenção Beyond The Wall não houve impacto significativo nas variáveis investigadas, já o Programa Silence is Deadly apresentou resultados promissores, porém investigou apenas o grupo experimental. O Programa SafeTALK é considerado eficaz quando executado em pequenos grupos.

Portanto, faz-se necessário implantar e implementar programas e políticas de prevenção do suicídio e da autolesão voltados para o público adolescente especificamente nos ambientes escolares, considerando o atual cenário epidemiológico mundial de aumento dos referidos comportamentos.

\section{Referências}

Aratangy E. W., Russo F. L., Giusti, J, S., Cordás, T.A. (2018). Como lidar com a automutilação: guia prático para familiares, professores e jovens que lidam com o problema da automutilação. Hogrefe

Benefield, L. E. (2003). Implementing evidence-based practice in home care. Home Healthc Nurse, 21(12), 804-811

Brito, M. D. L. de S., Silva Júnior, F. J. G. da, Costa, A. P. C., Sales, J. C. e S., Gonçalves, A. M. de S., \& Monteiro, C. F. de S. (2020). Comportamento suicida e estratégias de prevenção sob a ótica de professores. Escola Anna Nery, 24(4), 1-7. https://doi.org/10.1590/2177-9465-ean-2020-0109

Calear, A. L., Morse, A. R., Batterham, P. J., Forbes, O., \& Banfield, M. (2021). Silence is Deadly: A controlled trial of a public health intervention to promote help-seeking in adolescent males. Suicide and Life-Threatening Behavior, 51(2), 274-288. https://doi.org/10.1111/sltb.12703 
Carrol, J. C.; Talbot, Y., Permaul, J., Tobin, A., Moineddin, R., Blaine, S., Bloom, J., Butt, D., Kay, K., \& Telner, D. (2016). Academic family health teams: Part 1: patient perceptions of core primary care domains. Canadian Family Physician, 62(1), 23-30

Cervantes, C. A. D., \& Contreras, M. L. (2019). Intento de suicidio en adolescentes: Factores asociados. Revista chilena de pediatria, 90 (6), 606-616

Chang, E. C. (2017). Hope and hopelessness as predictors of suicide ideation in Hungarian college students. Death Studies 41(7), 455-460. $10.1080 / 07481187.2017 .1299255$

Eisenstein, C., Zamperoni, V., Humphrey, N., Deighton, J., Wolpert, M., Rosan, C., Bohan, H., A. Kousoulis, A., Promberger, M., \& Edbrooke-Childs, J. (2019). Evaluating the Peer Education Project in secondary schools. Journal of Public Mental Health, 18(1), 58-65. https://doi.org/10.1108/JPMH-07-20180048

Fernandes, E. S. F., \& Santos, A. M. dos. (2020). Desencontros entre formação profissional e necessidades de cuidado aos adolescentes na Atenção Básica à Saúde. Interface - Comunicação, Saúde, Educação, 24, 1-20. https://doi.org/10.1590/interface.190049

Gijzen, M. W. M., Creemers, D. H. M., Rasing, S. P. A., Smit, F., \& Engels, R. C. M. E. (2018). Evaluation of a multimodal school-based depression and suicide prevention program among Dutch adolescents: Design of a cluster-randomized controlled trial. BMC Psychiatry, 18(1), 1-12. https://doi.org/10.1186/s12888-018-1710-2

Giusti, J. S. Automutilação: características clínicas e comparação com pacientes com transtorno obsessivo-compulsivo. (2013). Tese, Psiquiatria, Universidade de São Paulo

Guedes, E. A. A., Mendes, M. LM., \& Messias, C. M. B. O. (2019). Práticas interdisciplinares de educação em saúde e meio ambiente na educação de jovens e adultos: percepção dos alunos. Revista Ibero-Americana de Estudos em Educação, 14(3), 1030-1048. 10.21723/riaee.v14i3.10064

Halicka, J., \& Kiejna, A. (2018). Non-suicidal self-injury (NSSI) and suicidal: Criteria differentiation. Advances in Clinical and Experimental Medicine, 27(2), 257-261. https://doi.org/10.17219/acem/66353

Kahn, P. J., Cohen, F. R., Tubiana, A., Legrand, K., Wasserman, C., Carli, V., Apter, A., Balazs, J., Banzer, R., Baralla, F., Barzilai, S., Bobes, J., Brunner, R., Corcoran, P., Cosman, D., Guillemin, F., Haring, C., Kaess, M.., Bitenc, U. M., Mészàros, G., McMahon, E., Postuvan, V., Saiz, P., Varnik, A., Varnik, P., Sarchiapone, M., Hoven, C. W. \& Wasserman, D. (2020). Infuence of coping strategies on the eficacy of YAM (Youth Aware of Mental Health): a universal school-based suicide preventive program. European Child \& Adolescent Psychiatry, 29 (12). https://doi.org/10.1007/s00787-020-01476-w

Kim, H. Y., Nam, E. W., Jin, K. N., \& So, A. Y. (2020). Effectiveness of a school-based mental health education program in an impoverished urban area of Peru. Global Health Promotion, 27(1), 77-86. https://doi.org/10.1177/1757975918786728

Kinchin, I., Russell, A. M. T., Petrie, D., Mifsud, A., Manning, L., \& Doran, C. M. (2020). Program Evaluation and Decision Analytic Modelling of Universal Suicide Prevention Training (SafeTALK) in Secondary Schools. Applied Health Economics and Health Policy, 18(2), 311-324. https://doi.org/10.1007/s40258-019-00505-3

Klim-Conforti, P., Zaheer, R., Levitt, A. J., Cheung, A. H., Schachar, R., Schaffer, A., Goldstein, B. I., Fefergrad, M., Niederkrotenthaler, T., \& Sinyor, M. (2021). The Impact of a Harry Potter-Based Cognitive-Behavioral Therapy Skills Curriculum on Suicidality and Well-being in Middle Schoolers: A Randomized Controlled Trial. Journal of Affective Disorders, 286(January), 134-141. https://doi.org/10.1016/j.jad.2021.02.028

Lima, A. L. O., Santos, B. F. T., Almeida, G. L., Ferreira, H. W., Mota, C. P., Messias, C. M., \& Silva, J. L. L. (2019). Educação em saúde mental no ambiente escolar: relato de caso. Saúde Coletiva (Barueri), 9(50)

Lindow, J. C., Hughes, J. L., South, C., Minhajuddin, A., Gutierrez, L., Bannister, E., Trivedi, M. H., \& Byerly, M. J. (2020). The Youth Aware of Mental Health Intervention: Impact on Help Seeking, Mental Health Knowledge, and Stigma in U.S. Adolescents. Journal of Adolescent Health, 67(1), 101-107. https://doi.org/10.1016/j.jadohealth.2020.01.006

Mansour, M., Krishnaprasadh, D., Lichtenberger, J., \& Teitelbaum, J. (2020). Implementing the Patient Health Questionnaire Modified for Adolescents to improve screening for depression among adolescents in a Federally Qualified Health Centre. BMJ Open Quality. doi:10.1136/bmjoq-2019-000751

Mo, P. K. H., Ko, T. T., \& Xin, M. Q. (2018). School-based gatekeeper training programmes in enhancing gatekeepers' cognitions and behaviours for adolescent suicide prevention: A systematic review. Child and Adolescent Psychiatry and Mental Health, 12(1), 1-24. https://doi.org/10.1186/s13034-018$0233-4$

Nasution, R. A., Keliat, B. A. \& Wardani, I. Y. (2019). Effect of cognitive behavioral therapy and peer leadership on suicidal ideation of adolescents in Bengkulu. Comprehensive Child And Adolescent Nursing, 42(sup1), 90-96. https://doi.org/10.1080/24694193.2019.1578300

Neves, C. C. S., Pereira, A. P. C. \& Pereira, C. A. S. (2020). Estratégias de prevenção do suicídio na escola para adolescentes: uma revisão de literatura na base medline. Research, Society and Development, 9(7), 1-21. http://dx.doi.org/10.33448/rsd-v9i7.3945

Persaud, S., Rosenthal, L., \& Arora, P. G. (2019). Culturally Informed Gatekeeper Training for Youth Suicide Prevention in Guyana: A Pilot Examination. In School Psychology International. 40(6), 624-640.

Polit, D. F., Beck, C. T. (2006). Using research in evidence-based nursing practice. In D. F. Polit, C. T. Beck (Eds.), Essentials of nursing research. Methods, appraisal and utilization (pp. 457- 494). Philadelphia

Quesada, A. A., Neto, C. H. A., Oliveira, J. M., Garcia, M. S. (2020). Automutilação: abordagem prática de prevenção e intervenção, Fundação Demócrito Rocha

Rasmussen, M. L., Haavind, H., \& Dieserud, G. (2018). Young Men, Masculinities, and Suicide. Archives of Suicide Research, 22(2), 327-343. https://doi.org/10.1080/13811118.2017.1340855 
Roberts, C. M., Kane, R. T., Rooney, R. M., Pintabona, Y., Baughman, N., Hassan, S., Cross, D., Zubrick, S. R., \& Silburn, S. R. (2018). Efficacy of the Aussie Optimism Program: Promoting Pro-social Behavior and Preventing Suicidality in Primary School Students. A Randomised-Controlled Trial. Frontiers in Psychology, 8 (February), 1-13. https://doi.org/10.3389/fpsyg.2017.01392

Severian, P. F. G., Melo, H. E., Eid, L. P., Gueroni, L. P. B., Souza, M.R., \& Pompeo, D. A. (2021). Impacto de intervenção psicoeducacional na autoeficácia. Revista de Enfermagem UERJ, 29. http://dx.doi.org/10.12957/reuerj.2021.53845

Silva, C. R., Cardinalli, I., Silvestrini, M. S., Farias, A. Z., Prado, A. C. S. A., Ambrosio, L., Oliveira, M. T., Paula, \& B. M. (2017). La terapia ocupacional y la cultura: miradas a la transformación social. Revista Chilena Terapia Ocupacional, 17(1), 105-113

Silva, L. (2019). Suicide among children and adolescents: a warning to accomplish a global imperative. Acta Paulista de Enfermagem, 32(3), 111-116. doi: https://10.1590/1982-

Sousa, C.M. S, Mascararenhas, M.D.M., Gomes, K.R.O, Rodrigues, M.T.P, Miranda, S. E. S., Frota, K.M.G.( 2020) Ideação suicida e fatores associados entre escolares adolescentes. Rev. Saúde Pública [online], 54 (33). https://doi.org/10.11606/s1518-8787.2020054001637

Souza, M. T. D., Silva, M. D. D. \& Carvalho, R. D (2010). Integrative review: what is it? How to do it?; Revisão integrativa: o que é e como fazer. Einstein (São Paulo), 8 (1)

Stewart, K., Martin, C., \& Fleming, M. (2018). Preventing suicide; nurse education and the occluded issue of gender. Nurse Education in Practice, 58-63. 10.1016/j.nepr.2018.07.004

Terpstra, S., Beekman, A., Abbing, J., Jaken, S., Steendam, M., \& Gilissen, R. (2018). Suicide prevention gatekeeper training in the Netherlands improves gatekeepers' knowledge of suicide prevention and their confidence to discuss suicidality, an observational study. BMC Public Health, 18(1), 1-8. https://doi.org/10.1186/s12889-018-5512-8

Testoni, I., Tronca, E., Biancalani, G., Ronconi, L., \& Calapai, G. (2020). Beyond the wall: Death education at middle school as suicide prevention. International Journal of Environmental Research and Public Health, 17(7). https://doi.org/10.3390/ijerph17072398Whittemore, R. \& Knafl, K. (2005). The integrative review: updated methodology. Journal Advanced Nursing, 52(5), 546-553

Tomek, S., Burton, S., Hooper, L. M., Bolland, A., \& Bolland, J. (2018). Suicidality in Black American Youth Living in Impoverished Neighborhoods: Is School Connectedness a Protective Factor? In School Mental Health. 10(1), 1-11.

Totura, C. M. W., Kutash, K., Labouliere, C. D., \& Karver, M. S. (2019). Evaluating Active Parental Consent Procedures for School Programming: Addressing the Sensitive Topic of Suicide Prevention. In Journal of School Health. 87(2), 114-120.

World Health Organization. (2014). Preventing suicide: a global imperative. World Health Organization

World Health Organization. (2018). Saúde Mental do Adolescente. Geneve: World Health Organization

Wyman, P. A., Pickering, T. A., Pisani, A. R., Rulison, K., Schmeelk-Cone, K., Hartley, C., Gould, M., Caine, E. D., LoMurray, M., Brown, C. H., \& Valente, T. W. (2019). Peer-adult network structure and suicide attempts in 38 high schools: implications for network-informed suicide prevention. Journal of Child Psychology and Psychiatry and Allied Disciplines, 60(10), 1065-1075. https://doi.org/10.1111/jcpp.13102

Zachariah, B., de Wit, E. E., Bahirat, J. D., Bunders-Aelen, J. F. G., \& Regeer, B. J. (2018). What Is in It for Them? Understanding the Impact of a "Support, Appreciate, Listen Team" (SALT)-Based Suicide Prevention Peer Education Program on Peer Educators. In School Mental Health. 10(4), 462-476. 